\title{
Pathopsychological features of cognitive style and cognitive errors of patients with repeated episodes of depression in manic-depressive disease
}

\author{
Vladimir Romek ${ }^{1 *}$,Evgenij Antokhin ${ }^{2}$, Rozalia Palaeva ${ }^{2}$, and Mikhail Maskov² \\ ${ }^{1}$ Don State Technical University, Faculty "Psychology, Pedagogy and Defectology", \\ 344000 Rostov-on-Don, Russian Federation \\ ${ }^{2}$ Orenburg State Medical University, Orenburg, Russian Federation
}

\begin{abstract}
In this work, a scientific and theoretical analysis of psychopathological features of depression patients was carried out, general concepts of manic-depressive disease (MDD), pathopsychological features of cognitive style, thinking errors were revealed. Pathopsychological processes in patients with recurrent depressive disorder (RDD) have been studied. Based on the results, recommendations on psycho-correction work with RDD patients are given.
\end{abstract}

\section{Introduction}

The relevance of the topic and the presentation of the problem of the present work is due to the lack of study of the pathopsychological features of mental processes in RDD within the framework of the MDD. Every year about 100 million inhabitants of our planet show signs of depression and accordingly need adequate medical care [1,2]. As one of the most clinically significant factors contributing to the increase in the frequency of depression, along with the process of urbanization, stressful events, increase in life expectancy, migration and other social trends, the more complete detection of depression in MDD, especially in patients of the general medical network, is considered. Such progress has been made possible by improving diagnostics using standardized criteria that ensure harmonization of clinical evaluation of the pathology studied [2]. At the same time, the dynamics of statistical indicators is affected by deepening perceptions of depressive spectrum disorders belonging to its "soft" pole and including erased, atypical forms - masked, somatised depressions, often comorbid somatic pathology [1].

It must be emphasized that depression has a number of adverse effects, both medical and social. In many cases of affective disease, the prolonged course or frequent recurrence of affective attacks, separated by incomplete (timopathic) remissions with persistent hypothemia, despite relatively shallow levels of psychopathological manifestations,

\footnotetext{
* Corresponding author: romekvova@gmail.com
} 
increases the risk of suicide, often committed during depression. The risk of suicide in combination of depression with chronic somatic or neurological disease is particularly high. Depression seriously affects the patient 's quality of life and adaptation capabilities, as it can lead to a decline in professional status with forced job changes, family breakdown and finally to complete disability $[1,2]$. Pathopsychological processes in depression are characterized by difficulty of concentration and concentration of attention, lack of memory, prevalence of dark, negative thoughts about themselves, about their life, about the world as a whole, pessimistic vision of the future with no perspective $[3,4,5]$. All this makes it very important to study in depth the causes leading to depressive conditions in modern society, indicates the priority of the chosen direction of the study. Its results, in our opinion, will contribute to more effective study of depressive conditions, as well as their diagnosis and creation of an optimal system of psychological adaptation to stressful conditions in the conditions of modern life.

The purpose of this survey is to study the pathopsychological features of cognitive style and thinking errors of RDD patients in MDD. In accordance with the purpose of the survey, tasks were formulated: 1. Conduct a theoretical analysis on the subject of the survey; 2 . Identify pathopsychological features of mental processes (cognitive style, cognitive errors) in RDD patients within MDD; 3. Identify differences in the nature of mental processes in patients with repeated episodes of depression compared to first-time MDD and postpsychotic depression (PPD) patients in the first episode of schizophrenia; 4. To develop recommendations for correction of depressive state of patients with MDD.

\section{Materials and methods}

Methods are chosen clinical-psychological (The Hamilton Rating Scale for Depression, The Hamilton Anxiety Rating Scale), experimental and psychological (The Stroop effect test, The Beck Cognitive Errors Questionnaire), statistical (nonparametric criterion of Mann-Whitney and methods of descriptive statistics). The sample consists of 44 patients with depressive disorder in MDD: 21 patients with a repeated episode of depression (RDD, main group) and 23 with a single depressive episode (first comparison group), as well as an additional sample of 23 patients with PDP in first psychotic epizode (second comparison group). The age of all patients is 18 to 50 years. Statistical processing was carried out using the standard software package for statistical mathematical calculation STATISTIKA 8. The statistical analysis did not reveal reliable differences in the social-demographic characteristics of the subjects, thus, in terms of education, sex, age, labour adaptation and number of attacks, the samples were comparable. Thus, the samples are comparable in social-demographic parameters (including age ranges - statistically significant differences: U-criterion $-\mathrm{p}=0.22$ ).

The use of clinical-psychopathological method consisted in diagnosis by a psychiatrist, analysis of archival documentation, as well as use of the The Hamilton Rating Scale for Depression and Anxiety Rating Scale, and in patients with schizophrenia - Calgary depression shizophrenia scale (CDSS) in order to detect the presence of anxiety and depression in the examined patients.

\section{Results}

By examining the cognitive style of thinking, a statistically significant difference is obtained from the scales of rigidity/flexibility and verbality/way of thinking (see Table 1). From Table 1 , it can be seen that the test subjects of the main group show a marked rigidity of cognitive control (69.13+44.22). This cognitive style characterizes the degree of subjective difficulty of patients with RDD in changing ways of processing information in situations of cognitive conflict. Rigid control indicates difficulties in moving from verbal functions to sensory- 
perceptual due to their low degree of automation. High interference rates in RDD patients suggest that these patients are dominated by a narrow, rigid cognitive style, weak automation of cognitive functions, and there is also an inability to slow down stronger verbal functions for the sake of color perception. Patients with a repeated depressive episode struggle to overcome cognitive conflict due to the need for special mental efforts to "separate" their verbal and sensory reactions. In rigid control, patients tend to overestimate external circumstances, while patients with a primary episode of depression, on the contrary, are more versatile in assessing the external situation. These data also indicate that RDD patients require more attention and conscious effort to automate any action. The rigidity of the cognitive style of patients with RDD may be due to the jamming of patients on the understanding of the negative sides of the situation and on their own negative emotions, which is typical of depressive thinking.

Patients with the first episode of schizophrenia have a less rigid style of thinking than patients with chronic depression, indicating more pronounced subjective difficulties of patients of the main group when changing ways of processing information in situations of cognitive conflict, i.e. when moving from verbal functions to sensory-perceptual. Consequently, PDP patients are less likely than RDD patients to be unable or unwilling to change the planned pattern of activities in circumstances where a previously intended program requires significant changes. The data obtained from the verbality index of the three test groups (see Table 1) show that the patients of the main group have a large expression of this factor, which indicates the prevalence among patients with chronic depression of the verbal method of processing information, i.e. their dominance of the speed of the word reading process over the process of calling colors. Subjects of this group require more time and attention to process sensoryperceptual stimulus, as such patients have a more developed ability to transmit their thoughts "in words." The statistically significant difference was obtained on the scale of verbality, which is more pronounced in the main group, a fact that also indicates that the subjects of the group require more attention to process sensory-perceptual information than verbal information. Thus, it is possible to speak about the prevalence of verbal method of information processing over sensory-perceptual among patients with RDD in MDD, therefore, in interpersonal communication they interact by means of speech, and patients with PPD in first psychosis epizode are guided by their own sensations and perception. In patients with schizophrenia, the dominant is the first signal system responsible for direct reflection of reality in the form of sensations and perception, and in RDD - the second, more mature, responsible for reflection of reality in the speech, verbal - sign form. The fact that schizophrenia patients are dominated by a sensory way of processing information can be due to personality destruction. In such patients, hypofrontality is more pronounced, i.e., frontal control is reduced, therefore, perception of environmental reality is distorted, control and regulation of behavior is impaired, as they are oriented to sensations and perception, so that patients with schizophrenia are less socially adapted than patients with RDD.

Table 1. Cognitive style indicators (The Stroop effect test)

\begin{tabular}{|l|l|l|l|}
\hline Indicator & $\begin{array}{l}\text { Main group } \\
(\mathbf{M} \pm \sigma)\end{array}$ & $\begin{array}{l}\text { Group of } \\
\text { comparison 1 } \\
(\mathbf{M} \pm \sigma)\end{array}$ & $\begin{array}{l}\text { Group of } \\
\text { comparison } \\
\mathbf{2}(\mathbf{M} \pm \sigma)\end{array}$ \\
\hline Rigidity $\backslash$ flexibility & $\mathbf{6 9 , 1 3} \pm \mathbf{4 4 , 2 2 *}$ & $\mathbf{4 9 , 2 9} \pm \mathbf{2 9 , 2 2 *}$ & $62,48 \pm 26,2$ \\
\hline Verbalnostlfigurativeness & $\mathbf{1 , 3 1 \pm 0 , 2 3 *}$ & $\mathbf{1 , 4 6} \pm \mathbf{0}, 25 *$ & $\mathbf{1 , 5 6} \pm \mathbf{0}, 3^{*}$ \\
\hline
\end{tabular}

Note: $*-\mathrm{p}<0.05, \mathrm{M}_{-}^{+\sigma}$ is the arithmetic mean of the standard deviation. 
Statistically significant differences in the Main group and in the Group of comparison 1 were revealed by the indicators of such cognitive errors as "splitting" (1.9 \pm 1.59$)$, "magnification/minimization" (1.2 \pm 0.89$)$, "disqualifying the positive" (2.3 \pm 1.29$)$, more pronounced in the main group of subjects (see Table 2). The tendency of patients with RDD to downplay their successes and underestimate positive experiences has been revealed. Patients with chronic depression, assessing themselves, another person or the situation, exaggerate or underestimate positive without proper grounds. Patients with RDD have a black-and-white vision of stressful situations, thinking in only two categories instead of many other options. Such patients tend to think extremes, divide events, people, acts into two opposite categories, in the absence of intermediate values. It's thinking characterized by maximalism. Speaking of himself, the patient usually chooses a negative category.

No statistically significant differences were found in the thinking errors of the main group and the second group of comparison of the subjects (see Table 2). It is also worth noting that patients of both groups have the most expressed thinking errors such as "emotional reasoning" and "disqualifying the positive" That is, both patients with the first episode of schizophrenia and patients with chronic depression have the conviction that if they experience some very strong emotion, that emotion is justified. It is a belief that something is only true because they "feel so" (essentially believe) it so strongly that they ignore or devalue evidence to the contrary. They are also prone to downplaying their successes, underestimating positive experiences.

In the test Group of comparison 2 more than in the Main group expressed such cognitive error as "labeling " $(1.3 \pm 1.27)$, which indicates that patients with first psychotic epizode give their actions or those of another negative or positive labels, which is most likely a manifestation of paranoia. In this group of patients also more clearly than in the Main group, there is a cognitive error called "splitting" $(2.1 \pm 1.24)$, which suggests a greater tendency of patients with first psychotic epizode to dichotomic, maximalist thinking, vision of stressful situations in black-and-white. Higher rates of "magnification/minimization" thinking error $(1.5 \pm 0.99)$ are also noted in this group of patients, indicating a baseless exaggeration or understatement of the positive when assessing yourself, person or situation.

In general, in the Main group of subjects higher rates by what in Groups of comparison $1 \& 2$ of the majority of cognitive errors, namely, "catastrophizing" are found (1.6 \pm 1.23$)$,

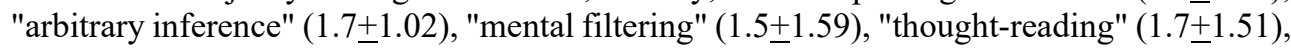

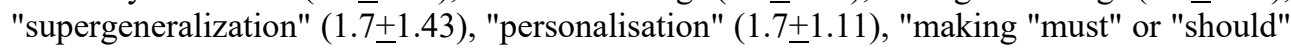

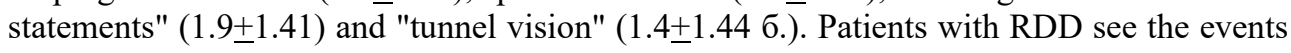
of their life exclusively in a negative light, life situations seem insurmountable to them, perceive everything that happens as unsustainable. They attribute to themselves responsibility for the conduct of others or for certain events and phenomena without taking into account more likely explanations, overestimate the extent to which events are related to them, and assume excessive responsibility. Patients with repeated episodes of depression draw conclusions even if they do not have reliable data about any situation or they clearly contradict these situations. More often, a general rule is drawn from one or more isolated cases or a conclusion is made that extends to a wide range of situations. This rule is beginning to apply, including to situations unrelated to it. Only what corresponds to their mood is perceived, although the perceived event can only be part of a much larger situation. They also have confidence that they know the thoughts, feelings, motives of others or those around them are able to know about their thoughts. RDD patients are more characteristic of having a clear, immutable idea of what other people should be and how they should behave and what their own behavior should be. If expectations are not met, a person perceives it as a failure. Also, RDD patients are more inclined to draw conclusions based on detail taken out of context while ignoring other, more substantial information. 
Cognitive evaluation of RDD patients approaches that of PPD patients in first psychotic epizode. In both groups of patients, there is a great connection between the emotional and cognitive spheres, hence the emotions of patients are determined by their thoughts. This may suggest that patients with RDD in MDD and PPE in first psychotic epizode appear to need fewer classes aimed at understanding cognitive patterns. On the basis of this, it is possible to talk about formation of a general group of psychotherapy for these groups of patients.

Table 2. Thinking Error Indicators (The Beck Cognitive Errors Questionnaire)

\begin{tabular}{|c|c|c|c|c|}
\hline № & Scale & $\begin{array}{l}\text { Main group } \\
\left(\mathbf{M}_{-} \sigma\right)\end{array}$ & $\begin{array}{l}\text { Group of } \\
\text { comparison } 1 \\
(\mathrm{M} \pm \sigma)\end{array}$ & $\begin{array}{l}\text { Group of } \\
\text { comparison } 2 \\
(M+\sigma)\end{array}$ \\
\hline 1 & Splitting & $1,9 \pm 1,59 *$ & $0,8 \pm 0,76 *$ & $2,1 \pm 1,24$ \\
\hline 2 & Catastrophizing & $1,6+1,23$ & $1,5 \pm 1,12$ & $1,1+1,04$ \\
\hline 3 & Arbitrary inference & $1,7+1,02$ & $1,6+1,1$ & $1,3+1,15$ \\
\hline 4 & $\begin{array}{l}\text { Disqualifying the } \\
\text { positive }\end{array}$ & $2,3 \pm 1,29 *$ & $1,3 \pm 0,95 *$ & $2,3 \pm 0,93$ \\
\hline 5 & Emotional reasoning & $2,2 \pm 1,38$ & $1,8 \pm 1,36$ & $2,3 \pm 0,96$ \\
\hline 6 & Labeling & $1,1+0,81$ & $1,1+0,94$ & $1,3+1,27$ \\
\hline 7 & $\begin{array}{l}\text { Magnification/minim } \\
\text { ization }\end{array}$ & $1,2 \pm 0,89 *$ & $0,3 \pm 0,73 *$ & $1,5 \pm 0,99$ \\
\hline 8 & Mental filtering & $1,5+1,59$ & $1,4+0,92$ & $1,4+1,20$ \\
\hline 9 & Thought-reading & $1,7 \pm 1,51$ & $1,2 \pm 1,24$ & $1,3 \pm 1,05$ \\
\hline 10 & Supergeneralization & $1,7 \pm 1,43$ & $0,9 \pm 0,85$ & $1,5 \pm 0,95$ \\
\hline 11 & Personalisation & $1,7 \pm 1,11$ & $1,2 \pm 1,24$ & $1,2 \pm 1,19$ \\
\hline 12 & $\begin{array}{l}\text { Making "must" or } \\
\text { "should" statements }\end{array}$ & $1,9 \pm 1,41$ & $1,5 \pm 0,98$ & $1,4 \pm 1,20$ \\
\hline 13 & Tunnel vision & $1,4 \pm 1,44$ & $1,2 \pm 0,1$ & $1,3 \pm 0,88$ \\
\hline
\end{tabular}

Note: * $-\mathrm{p}<0.05, \mathrm{M}+\sigma$ is the arithmetic mean of the standard deviation.

\section{Conclusion}

Thus, visible differences were revealed in the study of cognitive style and the prevailing way of processing information by patients. In patients with $\mathrm{RDD}$, the rigidity of cognitive control is more pronounced, which explains the fixation on their own negative emotions characteristic of depressive thinking and the jamming on the understanding of the negative sides of the situation. Patients with RDD have difficulty changing ways of processing information in situations of cognitive conflict. Rigid control also indicates difficulties in moving from verbal functions to sensory-perceptual due to their low degree of automation. Among patients with RDD, the prevalence of verbal processing of information is also noted.

In patients with depressive disorder, thinking is disadaptive, with thinking in patients in the main group being more shifted to the pole of disadaptivity, compared to sick comparison groups. Different cognitive errors have been found to prevail in different groups, but thinking errors are more pronounced and more common in the group of patients with repeated episodes of depression. For example, among patients with RDD, the most common cognitive errors are "disqualifying the positive" and "labeling" and among patients with a primary episode of depression, "emotional reasoning" and "arbitrary inference" This shows that patients with repeated episodes of depression are most characterized by a tendency to downplay their successes, underestimate positive experiences, and a tendency to endow themselves or others with unconditional, global characteristics without taking into account real facts. It can also 
be said that patients with RDD constantly adhere negative or positive labels to their actions or those of another person, and they themselves react acutely to the labels as if these labels are real things.

Patients with RDD in MDD have a less flexible thinking style than patients with PPD in first psychotic epizode. At the same time rigidity of cognitive style of patients with RDD is explained by the fact that depressive thinking is characterized by fixation on own negative emotions, on understanding of negative sides of the situation. The rigidity of the thinking style of patients with PPD is due to the fact that they struggle to overcome cognitive conflict due to the need for special mental efforts to "separate" their verbal and sensory reactions. The obtained data also indicate the prevalence of verbal method of information processing among patients with RDD (presence of frontal control).

There is a statistically significant difference in some cognitiveerrors in the Main group and in the Comparison group 1, with higher rates in the Main group, which reflects a greater degree of thought distortion in the group of patients with repeated depressive episodes compared to those with a single depressive episode. RDD patients and PPD patients in first psychotic epizode have no statistically significant differences in cognitive errors. In patients of both groups, cognitive errors such as "emotional reasoning" and " disqualifying the positive" are most pronounced. These data indicate that cognitive evaluation in depression patients with repeated episodes approaches that in patients with PPD in first psychotic epizode of schizophrenia. Taking into account the proximity of cognitive evaluation disorders (cognitive errors) of patients with RDD and PPD first psychotic epizode of schizophrenia in order to optimize the therapeutic process, it is possible to combine these patients into one group of cognitive therapy at the stage of copying-oriented training.

The work was published with the financial support of FGBU "Russian Foundation for Fundamental Research" (RFFR) within the framework of the scientific project "Development of a model of complex neuropsychological and psychophysiological assessment of suicidal risk and prediction of the development of affective disorders in adolescents and students" № 18-013-00015/19.

\section{References}

1. A. Smulevich, Depression in General Medicine: A Guide for Doctors (2007)

2. S. Obuhov, Psychiatry (2007)

3. A. Goryunov, J. of Neuropathology and Psychiatry name of S.S. Korsakov, 11 26-30 (2002)

4. B. Iovlev, E. Karpova, Rev. of psychiatry and med. psychology, 2 9-12 (1998)

5. V. Krasnov, Affective disorders (2011) 